\title{
Thermal vs quantum decoherence in double well trapped Bose-Einstein condensates
}

\author{
L. Pitaevskii ${ }^{a, b}$ and S. Stringari ${ }^{a}$ \\ ${ }^{a}$ Dipartimento di Fisica, Università di Trento, \\ and Istituto Nazionale per la Fisica della Materia, \\ I-38050 Povo, Italy \\ ${ }^{b}$ Kapitza Institute for Physical Problems, \\ ul. Kosygina 2, 117334 Moscow, Russia
}

(October 22, 2018)

\begin{abstract}
The quantum and thermal fluctuations of the phase are investigated in a cold Bose gas confined by a double well trap. The coherence of the system is discussed in terms of the visibility of interference fringes in both momentum and coordinate space. The visibility is calculated at zero as well as at finite temperature. The thermal fluctuations are shown to affect significantly the transition from the coherent to the incoherent regime even at very low temperatures. The coherence of an array of multiple condensates is also discussed.
\end{abstract}

After the first interference measurements carried out on two expanding and overlapping condensates [1] the problem of the relative phase between Bose-Einstein condensates has stimulated extensive theoretical work in the last few years (see for example [2] and references therein) as well as new experimental attempts to point out Josephson like phenomena [3, 1 . A challenging question is the transition from a coherent to an incoherent regime associated with the increase of the fluctuations of the relative phase between condensates confined in different traps. The transition can be in principle controlled by changing the tunneling probability between the wells either by tuning the height and the width of the barrier or the number of atoms. Most of theoretical works have focused so far on the ideal case of zero temperature where the fluctuations of the phase are of quantum nature. These works have shown that if the tunneling probability is sufficiently small the ground state of an atomic gas interacting with repulsive forces is not globally coherent, but exhibits new features of quantum nature, associated with the appearance of number squeezed configurations (see for example [5]).

The purpose of this letter is to discuss the relative importance of the quantum and thermal fluctuations in driving the transition between the coherent and incoherent regimes. Due to the low energy scale associated with the Josephson oscillation of the condensates the thermal effect may actually become crucially important even if one works at very low temperature.

Let us consider a dilute gas of atoms confined by an external potential characterized by a double well along the $x$ direction. The problem of an array of multiple wells will be discussed in the last part of the work. Near the bottom of the two traps the potential can be approximated by a harmonic function characterized by the oscillator frequency $\omega_{h o}$ which provides the relevant frequency scale of the collective excitations of each condensate (actually, due to the 3D nature of the problem, the harmonic expansion will in general introduce three different frequencies). We start our discussion by recalling the classical problem of the Josephson oscillation in the framework of the Gross-Pitaevskii (GP) theory for the order parameter. If the overlap between the condensates confined in the two wells is small we can naturally construct an approximate solution of the time dependent GP equation in the form

$$
\Psi(\mathbf{r}, t)=\left[\Psi_{l}\left(\mathbf{r}, N_{l}\right) e^{i \Phi_{l}}+\Psi_{r}\left(\mathbf{r}, N_{r}\right) e^{i \Phi_{r}}\right],
$$

where $\Psi_{l, r}\left(\mathbf{r}, N_{l, r}\right)$ are real functions satisfying the stationary GP equation in the left $(l)$ and right $(r)$ wells respectively and normalized to $\int d \mathbf{r} \Psi_{l, r}^{2}=N_{l, r}$ with $N_{l}+N_{r}=N$. The time dependence of the solution (11) is contained in the phases $\Phi_{l, r}$ and in the number of atoms $N_{l, r}$ confined in each well. The equations of motion can be written in the canonical form $\partial \Phi / \partial t=\partial H_{J} / \partial(\hbar k)$ and $\partial(\hbar k) / \partial t=-\partial H_{J} / \partial \Phi$ where

$$
H_{J}=\frac{1}{2} E_{C} k^{2}-E_{J} \cos \Phi .
$$

is the so called Josephson Hamiltonian [6, -7] depending on the conjugate variables $\hbar k$ and $\Phi$ where $k=\left(N_{l}-N_{r}\right) / 2$ is determined by the relative number of atoms in the two condensates and $\Phi=\Phi_{l}-\Phi_{r}$ is their relative phase. In deriving eq.(2) we have assumed $k \ll N$ and introduced the relevant parameters $E_{c}=2 d \mu_{l} / d N_{l}$ and $E_{J}=\left(\hbar^{2} / 2 m\right) \int d y d z\left[\Psi_{l} \partial \Psi_{r} / \partial x-\Psi_{r} \partial \Psi_{l} / \partial x\right]_{x=0}$ where $\mu_{l}$ is the chemical potential of each condensate and all the quantities are evaluated at $N_{l}=N_{r}=N / 2$. The term in $E_{c}$ accounts for the interaction effects in each condensate and vanishes in the non interacting gas since in this case the chemical potential does not depend on the number of atoms. In the Thomas-Fermi limit instead the parameter $E_{C}$ takes the value $E_{C}=(4 / 5) \mu_{l} / N_{l}$ due to the $N^{2 / 5}$ dependence of the chemical potential of a Bose-Einstein condensate trapped by a 3D harmonic potential. In the Thomas-Fermi regime the value of $E_{C}$ hence decreases with $N$. The term in $E_{J}$, which describes the tunneling probability between the two wells and is at the origin of the Josephson current, instead increases with $N$. Expression (2) for $H_{J}$ can be easily generalized to include the effects of gravity. 
Equation (11) represents a good approximation to the solution of the GP equation only if the Josephson motion is decoupled from the other modes of the condensate. This implies that the quantities $\Phi$ and $k$ should vary slowly in time with respect to the typical time $1 / \omega_{\text {ho }}$ characterizing the internal motion of each condensate. The Hamiltonian (2) corresponds to the classical problem of the pendulum. For small oscillations near equilibrium $(k=0, \Phi=0)$ the motion is of harmonic nature and is characterized by the classical plasma frequency

$$
\omega_{c l}=\frac{1}{\hbar} \sqrt{E_{J} E_{C}},
$$

so that, in order to ensure the decoupling from the internal oscillations of the condensate, the inequality $\omega_{c l} \ll \omega_{h o}$ should be satisfied.

Let us now proceed to quantize the classical Hamiltonian (2). This is achieved by replacing the conjugated variables $\Phi$ and $\hbar k$ with operators satisfying the commutation relation $[\hat{\Phi}, \hbar \hat{k}]=i \hbar$. It is convenient to work in the " $\Phi$-representation" where $\hat{k}=-i \partial / \partial \Phi$ and the quantum problem can be described by the Hamiltonian

$$
\hat{H}_{J}=-\frac{E_{C}}{2} \frac{\partial^{2}}{\partial \Phi^{2}}-E_{J} \cos \Phi
$$

acting in the space of the periodical functions of period $2 \pi$. The quantization introduces quantum fluctuations in the equilibrium state of the system whose nature depends on the ratio $E_{C} / E_{J}$. Because of the periodicity constraint the uncertainty relation obeyed by the fluctuations of $\Phi$ ad $k$ can be conveniently written in the form

$$
\left\langle\Delta k^{2}\right\rangle\left\langle\Delta \sin ^{2} \Phi\right\rangle \geq\langle\cos \Phi\rangle^{2} / 4
$$

where $\left\langle\Delta A^{2}\right\rangle=\left\langle\hat{A}^{2}\right\rangle-\langle\hat{A}\rangle^{2}$ is the variance of the general observable $\hat{A}$. The uncertainty relation (5) reduces to the usual form $\left\langle\Delta k^{2}\right\rangle\left\langle\Delta \Phi^{2}\right\rangle \geq 1 / 4$ only if the phase is localized around $\Phi=0$ [8]. The quantity

$$
\alpha=\langle\cos \Phi\rangle
$$

characterizing the right hand side of the uncertainty inequality (5) has a physical meaning of first importance. It provides the degree of coherence of the configuration and will be called the coherence factor. If the value of the phase is localized around zero, the value of $\alpha$ is close to unity (full coherence) and one can expand eq.(6) to calculate the quadratic fluctuations of the phase as $\left\langle\Delta \Phi^{2}\right\rangle=2(1-\alpha)$. If instead the phase is delocalized and all its values are equally probable, then the value of $\alpha$ is zero (absence of coherence).

The parameter $\alpha$ is directly related to measurable observables. For example if one calculates the in situ momentum distribution of two separated condensates with a fixed relative phase $\Phi$, one finds fringes in momentum space given by the expression [9] $n(\mathbf{p})=$ $2\left[1+\cos \left(p_{x} d / \hbar+\Phi\right)\right] n_{0}(\mathbf{p})$ where $n_{0}(\mathbf{p})$ is the momentum distribution of each condensate and $d$ is the relative distance along the $x$ axis. The typical width of $n_{0}(\mathbf{p})$ is given by $\hbar / R$ where $R$ is the spatial size of each condensate. The momentum distribution of a single trapped Bose gas has been already the object of measurements based on stimulated light scattering [10]. If the value of the relative phase fluctuates the average value of the momentum distribution at equilibrium is described by the equation

$$
n(\mathbf{p}))=2\left[1+\alpha \cos \left(p_{x} d / \hbar\right)\right] n_{l}(\mathbf{p})
$$

where the parameter $\alpha$ corresponds to the average $\langle\cos \Phi\rangle$ and hence coincides with the coherence factor introduced in eq.(6) (we have used the fact that $\langle\sin \Phi\rangle=0$ at equilibrium). One should not however confuse the parameter $\alpha$ with the visibility of fringes obtained in a single realization of the experiment which can give rise to a well defined interference pattern even if the initial state is not coherent. The parameter $\alpha$ corresponds instead to an average taken on several measurements and is consequently an intrinsic property of the state of the system. This distinction is crucial if one considers interference experiments with two condensates. A similar structure characterizes also the fringes of the density distribution measured after expanding the condensates [1]. In this case in order to obtain a simple result one has to make the additional assumption that the condensates do not interact each other when they overlap. For long expansion times $t$ the phases of the two condensates evolve according to the law $(\mathbf{r} \pm \mathbf{d} / 2)^{2} m / \hbar t$ [11] so that the full density profile takes the following asymptotic form

$$
n(\mathbf{r}, t))=\left[n_{+}+n_{-}+2 \alpha \cos \left(\frac{m d}{\hbar t} x\right) \sqrt{n_{+} n_{-}}\right]
$$

where $n_{ \pm}=n_{0}(\mathbf{r} \pm \mathbf{d} / 2, t)$ are the densities of the two expanding condensates. Eqs. (78 8) show that the value of $\alpha$ can be inferred from either the measurement of the momentum distribution and/or of the density profile after expansion, although in the second case the conditions of applicability of eq.(8) are more severe.

Let us now discuss the behaviour of the ground state of the Josephson Hamiltonian (4). In the limit of strong tunneling $E_{C} / E_{J} \ll 1$ the system undergoes small oscillations around the equilibrium value $\Phi=0$ and the Hamiltonian becomes quadratic in $\Phi$. In this limit the fluctuations of the phase are given by $\left\langle\Delta \Phi^{2}\right\rangle=$ $\left(\sqrt{E_{C} / E_{J}}\right) / 2 \ll 1$ and are hence small. One can consequently regard the inequality $E_{C} / E_{J} \ll 1$ as the physical condition for applying the classical GP theory to the problem of the double-well potential and, consequently, to treat the systems as a globally coherent object described by a unique order parameter. Also the fluctuations of $k$ in the ground state can be easily calculated and one finds the result $\left\langle\Delta k^{2}\right\rangle=\left(\sqrt{E_{J} / E_{C}}\right) / 2 \gg 1$. In 
the strong tunneling limit the uncertainty relation (5) reduces to the usual form $\left\langle\Delta k^{2}\right\rangle\left\langle\Delta \Phi^{2}\right\rangle \geq 1 / 4$ and actually becomes an identity as a consequence of the harmonic nature of the Hamiltonian. Notice, however, that if $\left\langle\Delta \Phi^{2}\right\rangle$ is of the order of $1 / N$ or smaller, the formalism developed above is no longer adequate and should be improved through both the inclusion of the factor $\sqrt{1-4 k^{2} / N^{2}}$ in the term $-E_{J} \cos \Phi$ of the Josephson Hamiltonian (4), and a more microscopic approach to the phase operator (see for example [12] and references therein). In the following we will mainly limit our discussion to the case $E_{C} \gg E_{J} / N^{2}$ which ensures that the quadratic fluctuations of the phase are in any case larger than $1 / N$.

In the case of weak tunneling $E_{C} / E_{J} \gg 1$ the behaviour of the fluctuations is very different. The Josephson term $E_{J}$ entering the Hamiltonian (4) can be neglected in first approximation and the wave function of the ground state is simply a constant given by $1 / \sqrt{2 \pi}$, showing that the relative phases between the two condensates is distributed in a random way. At the same time the fluctuation of the relative number $k$ of atoms in the two traps becomes smaller and smaller and vanish like $2\left(E_{J} / E_{C}\right)^{2}$. This is consistent with the uncertainty relation (5) since in the same limit the coherence factor vanishes like $\alpha \rightarrow 2\left(E_{J} / E_{C}\right)$. In the weak tunneling limit the ground state of the system exhibits fragmented Bose-Einstein condensation and is characterized by the macroscopic occupation of the two single-particle states localized in the two wells.

In fig. 1 we show the coherence factor $\alpha$ as a function of the ratio $E_{C} / E_{J}$ calculated by solving explicitly the Schrödinger equation for the ground state with the Josephson Hamiltonian (位. The figure shows that for values of $E_{J}$ smaller than $E_{C}$ the coherence factor is significantly quenched, pointing out the occurrence of a continuous transition to the number-squeezed regime. The transition is accompanied by a change of the lowest excitation energy $\omega_{p}$ of $\hat{H}_{J}$ from the classical value (3) to the "free" value $E_{C} / 2$ which is obtained by setting $E_{J}=0$ in (4). An accurate expression for $\omega_{p}$ is obtained by evaluating the ratio between the cubic and energy weighted sum rules $\left\langle\left[\left[\hat{k}, \hat{H}_{J}\right],\left[\hat{H}_{J},\left[\hat{H}_{J}, \hat{k}\right]\right]\right]\right\rangle=E_{J}^{2} E_{C}\left\langle\cos \Phi^{2}\right\rangle$ and $\left\langle\left[\hat{k},\left[\hat{H}_{J}, \hat{k}\right]\right]\right\rangle=E_{J}\langle\cos \Phi\rangle$, relative to the operator $\hat{k}$. The result is

$$
\omega_{p}=\frac{1}{\hbar} \sqrt{E_{J} E_{C} \frac{\left\langle\cos ^{2} \Phi\right\rangle}{\langle\cos \Phi\rangle}}
$$

where the averages should be evaluated on the ground state. In the limit $E_{C} \ll E_{J}$ one has $\left\langle\cos ^{2} \Phi\right\rangle=\langle\cos \Phi\rangle=$ 1 and one recovers the classical result (3). In the opposite limit one finds $\left\langle\cos \Phi^{2}\right\rangle=1 / 2,\langle\cos \Phi\rangle=2 E_{J} / E_{C}$ and $\omega_{p}=E_{C} / 2 \hbar$.

So far we have investigated the problem in an ideal situation at zero temperature. Due to the smallness of the plasma frequency (3) it is important to under- stand the role of the thermal fluctuations of the relative phase of the two-condensate system. These are expected to become important as soon as the temperature is of the order or higher than $\hbar \omega_{p}$. To investigate the thermal effect we have calculated the thermal average $\alpha(T)=\sum_{n} \alpha_{n} \exp \left[-E_{n} / T\right] / \sum_{n} \exp \left[-E_{n} / T\right]$ of the coherence factor where $n$ and $E_{n}$ are the eigenstates and eigenenergies of the Josephson Hamiltonian (雨), $\alpha_{n}=\langle n|\cos \Phi| n\rangle$ are the corresponding quantum averages and we have set the Boltzmann constant equal to 1 . The results are presented in fig. 2 as a function of the parameter $T / E_{J}$ for two different values of $E_{C} / E_{J}$. At $T=0$ one recovers the values of $\alpha$ given in fig.1. One clearly see that even if quantum effects are small the thermal decoherence of the phase becomes important at temperatures of the order $T \sim E_{J}$. The full line in the same figure gives the classical prediction

$$
\alpha_{c l}(T)=\frac{\int_{-\pi}^{+\pi} d \Phi \cos \Phi \exp \left[E_{J} \cos \Phi / T\right]}{\int_{-\pi}^{+\pi} d \Phi \exp \left[E_{J} \cos \Phi / T\right]}
$$

holding for $T \gg \omega_{p}$. If $T \ll E_{J}$, eq. (10) gives the result $\left\langle\Delta \Phi^{2}\right\rangle=T / E_{J} \ll 1$ for the classical fluctuation of the phase. The fluctuation of $k$ can be also easily calculated starting from the classical expression (2) and one gets the result $\left\langle\Delta k^{2}\right\rangle=T / E_{C}$ for any value of $T$. The thermal fluctuations of $k$ may be large and consequently at finite $T$ the system will not in general exhibit the phenomenon of number squeezing, even if the condition $E_{J} \ll E_{C}$ is satisfied.

It is useful to discuss the relevant scale of energies and temperatures in a specific example. Let us consider a system of $10^{3}$ atoms per well and the value $\mu=5 \hbar \omega_{h o}$ for the chemical potential. The coupling constant $E_{c}$ is about $5 \times 10^{-3} \hbar \omega_{h o}$. By choosing $E_{J}=\hbar \omega_{h o}$ one has $\omega_{p} \sim 0.1 \omega_{h o}$. With the above choices both the conditions $\omega_{p} \ll \omega_{h o}$ and $E_{C} \gg E_{J} / N^{2}$ needed to apply the formalism developed above are well satisfied. At $T=0$ the system is practically coherent $(\alpha \simeq 1)$ since $E_{C} \ll E_{J}$. However if $T$ is of the order of the oscillator energy $\hbar \omega_{h o}$, which corresponds to a rather low temperature in standard experiments, the coherence is partially lost because of thermal effects. By changing the value of $E_{J}$ and/or $T$ one can clearly explore other interesting regimes.

The Josephson effect with two condensates can be naturally extended to an array of multiple wells. This extension is particular relevant because of the recent experimental efforts to investigate one-dimensional optical lattices 4 . The array of condensates confined in multiple wells is described by the multiple-condensate Hamiltonian

$$
\hat{H}_{J}=-\frac{1}{4} E_{C} \sum_{k=1}^{N_{s}} \frac{\partial^{2}}{\partial \Phi_{k}^{2}}-E_{J} \sum_{k=1}^{N_{s}-1} \cos \left(\Phi_{k+1}-\Phi_{k}\right)
$$

where $N_{s}$ is the number of wells, $\Phi_{k}$ is the phase of the $k$-th condensate and $E_{C}, E_{J}$ are the parameters charac- 
terizing the double-well Hamiltonian (位. In the large $N_{s}$ limit the one-dimensional Hamiltonian (11) is known to exhibit a phase transition at zero temperature occurring at the critical value $E_{C}=1.62 E_{J}[13]$. The "superfluid" phase is not accompanied by the appearence of an order parameter and cannot be described in terms of mean field theories. It is instead characterized by an algebraic decay of the phase correlation function $\left\langle\cos \left(\Phi_{k}-\Phi_{l}\right)\right\rangle$ at large distances $|k-l| \gg 1$ (quasi long range order). The decay becomes exponential for $E_{C}>1.62 E_{J}$. The phase transition disappears at finite temperature. Actually in the "classical" regime of high temperatures the phase correlation functions can be evaluated analytically and one finds the simple result

$$
\left\langle\cos \left(\Phi_{k}-\Phi_{l}\right)\right\rangle_{c l}=\alpha_{c l}^{|k-l|}
$$

which exhibits an exponential decay for large values of $|k-l|$. In eq.(12) $\alpha_{c l}$ is the classical value (10) calculated for the double well problem. Result (12) can be used to evaluate the momentum distribution $n(\mathbf{p})$ of the array. By taking the Fourier transform $\Psi(\mathbf{p})=$ $(2 \pi \hbar)^{-3 / 2} \int d \mathbf{r} \Psi(\mathbf{r}) \exp [-i \mathbf{p} \cdot \mathbf{r} / \hbar]$ of the order parameter $\Psi(\mathbf{r})=\sum_{k=1}^{N_{s}} \Psi_{k}(\mathbf{r}) e^{i \Phi_{k}}$ and using the relationship $\Psi_{k}(\mathbf{p})=\Psi_{l}(\mathbf{p}) \exp \left[-i(k-j) p_{x} d / \hbar\right]$ following from the translational invariance properties of the array, one finds the result

$$
\begin{aligned}
n(\mathbf{p}) & =n_{0}(\mathbf{p}) \sum_{k, l} e^{-i(k-l) p_{x} d / \hbar}\left\langle\cos \left(\Phi_{k}-\Phi_{l}\right)\right\rangle \\
& =N_{s} n_{0}(\mathbf{p}) \frac{1-\alpha_{c l}^{2}}{1+\alpha_{c l}^{2}-2 \alpha_{c l} \cos \left(p_{x} d / \hbar\right)}
\end{aligned}
$$

where $n_{0}(\mathbf{p})$ is the momentum distribution of each condensate, $d$ is the distance between two consecutive condensates and, in deriving the last identity, we have assummed $N_{s} \gg 1\left(1-\alpha_{c l}\right)$. Result (14) explicitly points out the effects of coherence. When $\cos \left(p_{x} d / \hbar\right)=1$ the incoherent signal $N_{s} n_{0}(\mathbf{p})$ is amplified by the factor $\left(1+\alpha_{c l}\right) /\left(1-\alpha_{c l}\right)$. Viceversa, when $\cos \left(p_{x} d / \hbar\right)=-1$ the signal is suppressed by the factor $\left(1-\alpha_{c l}\right) /\left(1+\alpha_{c l}\right)$.

In conclusion we have investigated the consequences of the quantum and thermal fluctuations of the phase on the coherence phenomena exhibited by Josephson like configurations. Thermal effects are predicted to play a major role even at very low temperature and should be consequently taken into account in order to control the transition to the new quantum phases exhibited by these ultracold Bose gases.

Useful discussions with M. Inguscio, M.A. Kasevich and A. Smerzi are acknowledged. This research is supported by the Ministero della Ricerca Scientifica e Tecnologica (MURST).
[1] M.R. Andrews, C.G. Townsend, H.-J. Miesner, D.S. Durfee, D.M. Kurn, and W. Ketterle, Science 275, 637 (1997).

[2] A.J. Leggett, Rev. Mod. Phys., April 2001.

[3] D.S. Hall, M.R. Matthews, C.E. Wieman, and E.A. Cornell, Phys. Rev. Lett. 81, 1542 (1998).

[4] B.P. Anderson and M.A. Kasevich, Science 282, 1696 (1998); C. Orzel, A.K. Tuchman, M.L. Fensclau, M. Yasuda, and M.A. Kasevich, Science 291, 2386 (2001).

[5] J. Javanainen, M. Ivanov, Phys. Rev. A 60, 2351 (1999).

[6] A. Smerzi, S. Fantoni, S. Giovannazzi, and S.R. Shenoy, Phys. Rev. Lett., 79, 4950 (1997)

[7] I. Zapata, F. Sols, and A.J. Leggett, Phys. Rev. A, 57, R28 (1998)

[8] If the relative phase is localized around a value $\Phi_{0} \neq 0$ one can simply use (5) by replacing $\Phi$ with $\Phi-\Phi_{0}$

[9] L.P. Pitaevskii, S. Stringari, Phys. Rev. Lett. 83, 4237 (1999).

[10] J. Stenger, S. Inouye, A.P. Chikkatur, D.M. StamperKurn, D.E. Pritchard, and W. Ketterle, Phys. Rev. Lett. 82, 4569 (1999).

[11] F. Dalfovo, S. Giorgini, L.P. Pitaevskii, and S. Stringari, Rev. Mod. Phys. 71, 463 (1999).

[12] J.R Anglin, P. Drummond, and A. Smerzi, condmat/0011440

[13] R.M. Bradley and S. Doniach, Phys. Rev. B 30, 1138 (1984)

Fig.1 Coherence factor $\alpha$ at zero temperature as a function of the ratio $E_{C} / E_{J}$.

Fig.2 Coherence factor $\alpha$ as a function of the ratio $T / E_{J}$ for $E_{C} / E_{J}=3$ (a), $E_{C} / E_{J}=1$ (b). The classical curve $(10)$ is also shown (c). 


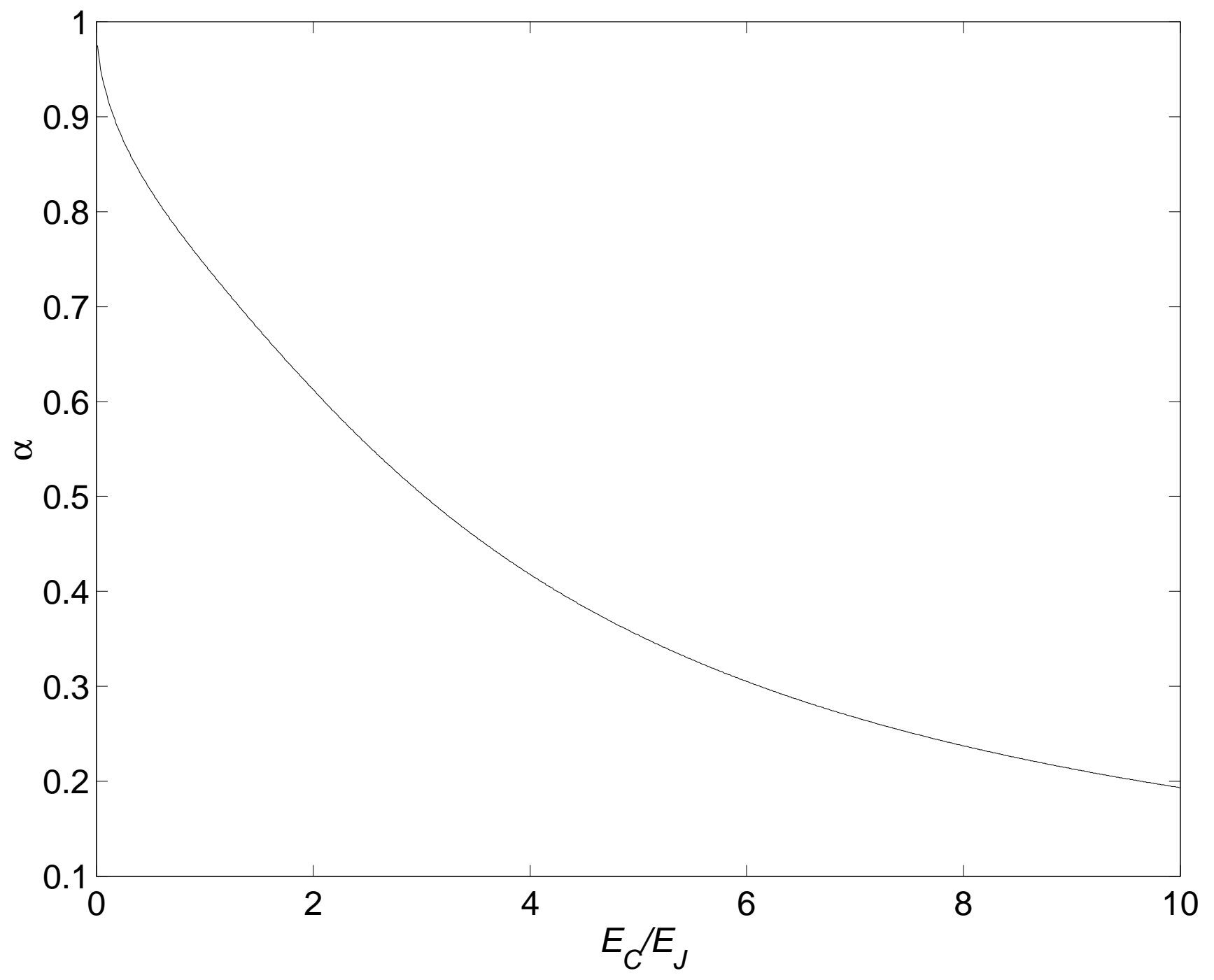




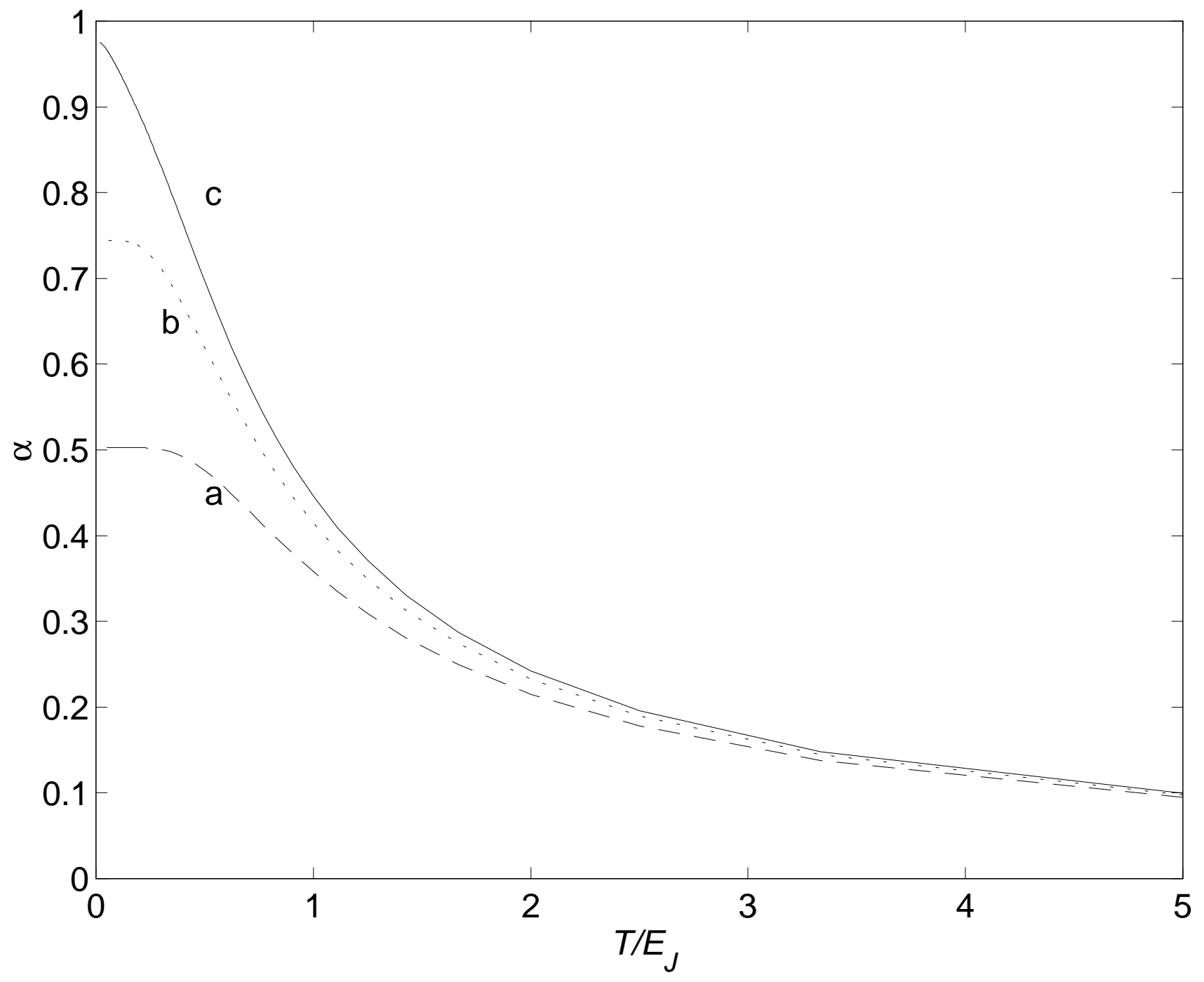

